\title{
Mixed conduction in charge transfer materials based on aromatic diamines and iodine having different mole ratios
}

\author{
RAM ADHAR SINGH* and VINOD KUMAR SINGH \\ Molecular Electronics Laboratory, Department of Chemistry, Faculty of Science, Banaras \\ Hindu University, Varanasi 221005 , India \\ MS received 12 June 1996; revised 11 February 1997

\begin{abstract}
Mixed ionic and electronic conduction in charge transfer materials based on aromatic diamine electron donors (benzidine, o-tolidine $\left(3,3^{\prime}\right.$-dimethyl benzidine) and $N, N^{\prime}$-diphenylbenzidine) with iodine in different mole ratios has been reported. The current-voltage and capacitance-voltage curves as a function of time and temperature have been obtained to determine the role of ionic conduction in electronic conductors based on charge transfer complexes. The compositional dependence of ionic transport numbers, diffusion parameters, ionic $\left(\sigma_{i}\right)$ and electronic $\left(\sigma_{\mathrm{e}}\right)$ conductivities and thermal activation energies has been studied. Temperature and frequency dependence of AC impedance and related parameters have been studied to learn about the electrical conduction behaviour in these non-stoichiometric charge transfer materials. An electrochemical mechanism has been proposed to account for the ionic conduction in some of these complexes.
\end{abstract}

Keywords. Charge transfer materials; mixed conduction; transport numbers; electrical conductivity.

\section{Introduction}

Charge transfer materials usually support electronic conduction (Gutmann and Lyons 1967; Ashwell 1992). The absence of any relaxation effects, i.e. the variation of current with time after applying a voltage, often found in the case of ionic conductors, has been used as an evidence for the electronic conduction in organic materials. The cases, where such time dependences were observed were accounted on the basis of space charge limited currents (Gutmann and Lyons 1967).

Surprisingly, in the electrical conductivity of perylene-bromine complex, a part of conductance was attributed to the transference of ions and ionic conductance was found to be 3-5 times the electronic conductance in the freshly prepared samples and 10-20 times after some time (Akamatu et al 1954). Thus, the first organic conductor exhibited ionic conduction along with electronic conduction. However, the argument given to account for the increase in resistivity was because of the chemical reaction of bromine with perylene or a small electromotive force of a few millivolts in the specimen cell. The sublimation of iodine because of heating at the contact points was believed to be the reason for this behaviour (Kommandeur and Hall 1961). However, this may not be correct in the light of formation of $\mathrm{AgBr}$ by the reaction of bromine vapour with the metallic cap made up of silver. This compound is an ionic conductor and observed ionic conduction may be due to this process (Chandra 1981).

The increase in resistivity of phenothiazine-iodine complex, using silver paint contacts, was recently observed and stable values were found when graphite was used as a contact (Singh et al 1991). Electronic conduction in the complexes of hydrocarbons

\footnotetext{
*Author to whom all correspondence should be addressed
} 
with iodine has been reported (Akamatu et al 1956). On passage of excess charge, insignificant change in conductivity indicated that conduction was electronic. Various non-stoichiometric compositions of $p$-phenylene diamine-iodine complexes were prepared and the ionic conduction was isolated by passage of dc current for $15 \mathrm{~h}$ (Nisizaki and Kusakawa 1963). However, the relative magnitude of ionic to electronic conduction has not been reported in this paper. The compositional dependence of electrical conductivity and activation energy for iodine complexes with various aromatic diamines show the minimum resistivity value at 1:1-25 molar ratios for aromatic diamines containing two benzene rings (Nisizaki and Kusakawa 1965).

Semiconduction in organic polyiodides results from the layers of iodine atoms, with $\mathrm{V}$-shaped pentaiodide ions so arranged as to form a nearly square net of iodine (Kusabayashi et al 1964). The formation of polyiodides in phenothiazine-iodine complex with stair-case like arrangement of iodine atoms has been studied by resonance Raman and Mössbauer spectroscopy (Sakai et al 1987). Non-ohmic conduction and electrical switching under pressure has been reported for $o$-tolidine-iodine (1:1) charge transfer complex (Naik and Subramanyam 1986). Marked deviations from ohmicity have been found, which were attributed to some form of electronic excitations in the system. Electrical switching and memory phenomena has been reported in benzidine-DDQ and $o$-tolidine-DDQ under pressure (Ravindran and Subramanyam 1991a, b). Field-induced phase transition has been proposed to be responsible for this behaviour.

The above conflicting views led us to study the time-dependence and temperaturedependence of electrical properties (both dc and ac) of some charge transfer complexes of aromatic diamines with iodine in which ionic conduction along with the electronic conduction has been found. We present a number of studies such as linear and cyclic current-voltage curve, their time dependence and temperature dependence along with their IR spectra before and after electrolysis to prove ionic as well as electronic conduction in these materials. Such materials are very useful for solid state battery. We have recently shown that rechargeable iodine batteries could be fabricated using charge transfer complexes of iodine with aromatic diamines (Singh et al 1996a, b).

\section{Experimental}

\subsection{Materials and methods}

Benzidine (Stains), o-tolidine (CDH), $N, N^{\prime}$-diphenylbenzidine (Sigma) and iodine (E. Merck) were purified by crystallization and sublimation. The complexes having different compositions were prepared by mixing appropriate amounts of the components dissolved in hot benzene solvent. The precipitates were filtered, washed twice with pure solvent and dried over anhydrous $\mathrm{CaCl}_{2}$. The compositions of samples were analysed by elemental analyses and iodometric titrations, which agreed well with the overall stoichiometric ratios of the complexes.

\subsection{Measurements}

The finely powdered charge transfer materials were made into pellet forms using hydraulic press (Spectralab) at a pressure of $10 \mathrm{Kbar}$. Current-voltage characteristics 
and conductivity measurements were performed as a function of time and temperature using a Source-Measure Unit(Keithley Model 236) and temperature controller (Century India Model CT 806). An AC impedance analyser (Keithley, USA, Model 3330 LCZ Meter) was used for measuring AC electrical properties as a function of frequency and temperature. The dielectric constant was calculated with the help of measured capacitance. All the measurements were repeated at least thrice to achieve reproducible values. FTIR spectra of samples were recorded in $\mathrm{KBr}$ in the $4000-600 \mathrm{~cm}^{-1}$ region on a Jasco (FTIR-5300) infrared spectrophotometer.

\section{Results and discussion}

\subsection{FTIR spectral studies}

Benzidine and its analogues form stable dications electrochemically (Weinberg 1974). Therefore, it may be possible to form their ionic charge transfer complexes by chemical oxidation using iodine. The formation of charge transfer complexes of aromatic diamine with iodine shows distinct changes in the infrared spectra of the complexes

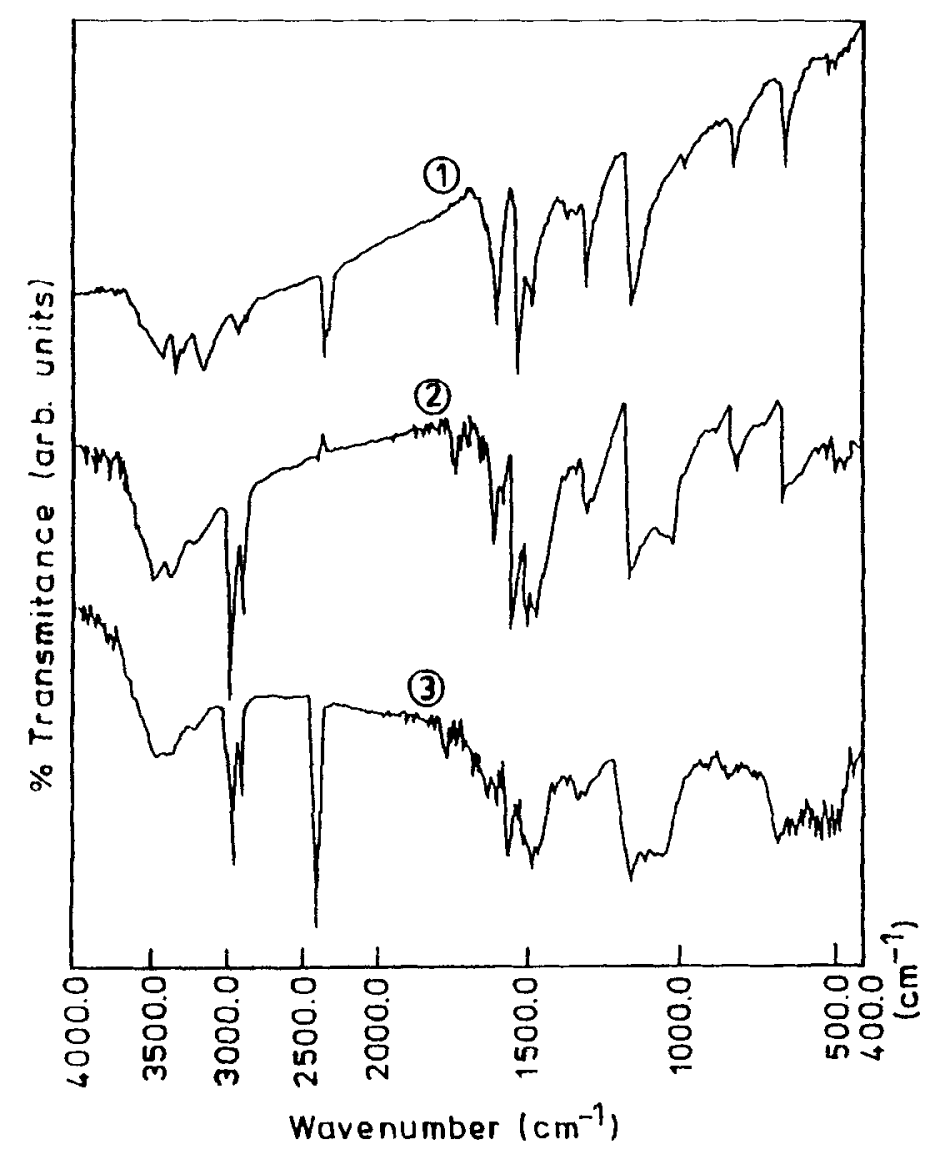

Figure 1. Infrared spectra of benzidine-iodine $(1: 1 \cdot 25)$ pure complex (1), from cathode side (2) and from anode side (3) of the pellet after electrolysis. 
having different compositions from their component spectra. Pronounced shifts in the IR spectra of different compositions have been observed. A strong $\mathrm{N}-\mathrm{H}$ stretching band at $3383.45 \mathrm{~cm}^{-1}$ shifts to $3352.58 \mathrm{~cm}^{-1}$ at $1: 0.75 \mathrm{Bz}-\mathrm{I}_{2}$ ratio, $3354.5 \mathrm{~cm}^{-1}$ at $1: 1$ $\mathrm{Bz}-\mathrm{I}_{2}$ ratio and $3314 \mathrm{~cm}^{-1}$ at $1: 1 \cdot 25 \mathrm{Bz}-\mathrm{I}_{2}$ ratio. Similarly $\beta\left(\mathrm{NH}_{2}\right)$ for pure benzidine at $1604.92 \mathrm{~cm}^{-1}$ changes to $1608.78,1606$ and $1604.92 \mathrm{~cm}^{-1}$ respectively for the above ratios. Such changes clearly indicate compositional dependence of molecular species present in various complexes. The broad bands at $\mathrm{N}-\mathrm{H}$ positions indicate formation of the quaternary amine species i.e. $\mathrm{N}^{+}-\mathrm{H}$ from which a labile proton is expected. The infra-red spectra of samples collected from the two faces of pellets after electrolysis (the cathode and anode side) have been included in figure 1 . These spectra are quite different from the complex before electrolysis, indicating changes in molecular structure on electrolysis. A possible mechanism for the formation of different species at the cathode and the anode during electrolysis may be proposed as follows:

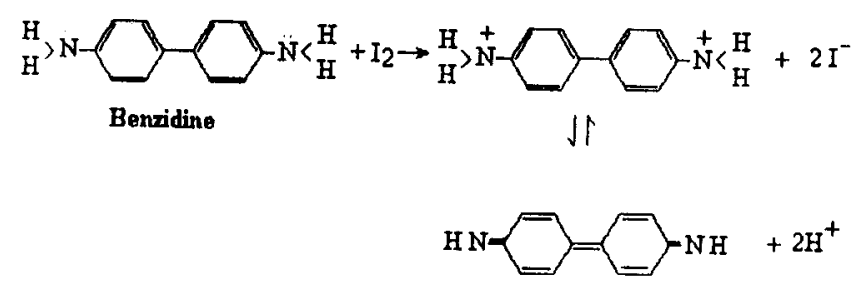

At cathode

$$
2 \mathrm{H}^{+}+2 \mathrm{e}^{-} \rightarrow \mathrm{H}_{2} \uparrow
$$

At anode

$$
2 \mathrm{I}^{-} \rightarrow 2 \mathrm{I}^{+} \rightarrow \mathrm{I}_{2}
$$

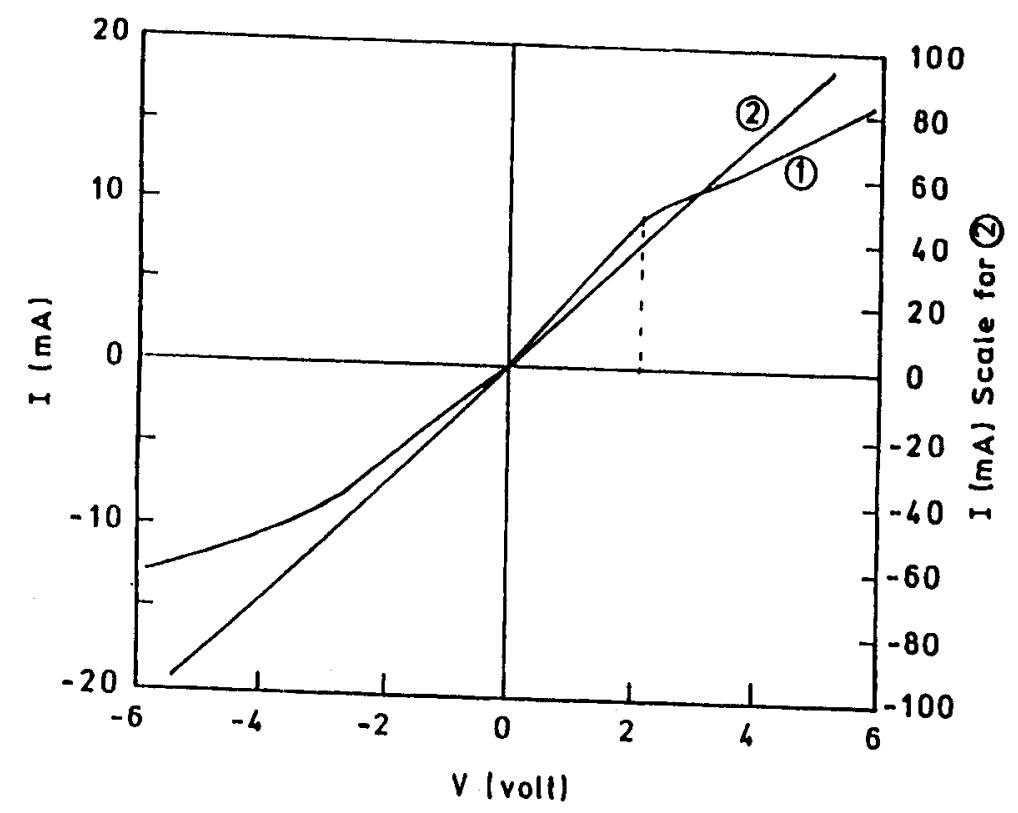

Figure 2. $I-V$ characteristics of benzidine-iodine $(1: 1 \cdot 25)$ (1) and $N, N^{\prime}$-diphenylbenzidine-iodine $(1: 1 \cdot 25)(2)$. 


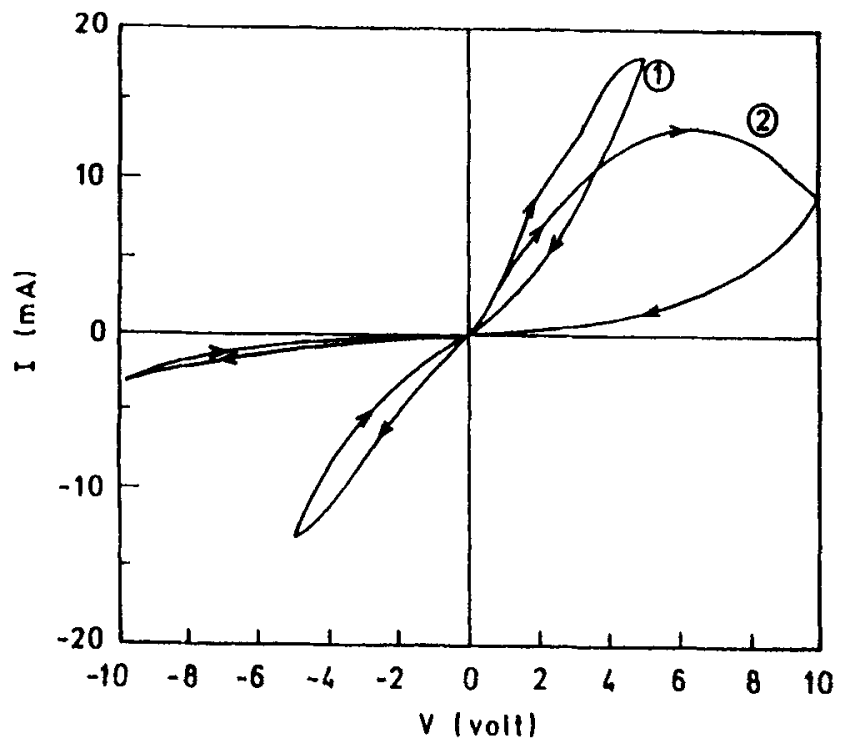

Figure 3. Cyclic $I-V$ for benzidine-iodine $(1: 1 \cdot 25)$ up to $4(1)$ and $10 \mathrm{~V}(2)$.

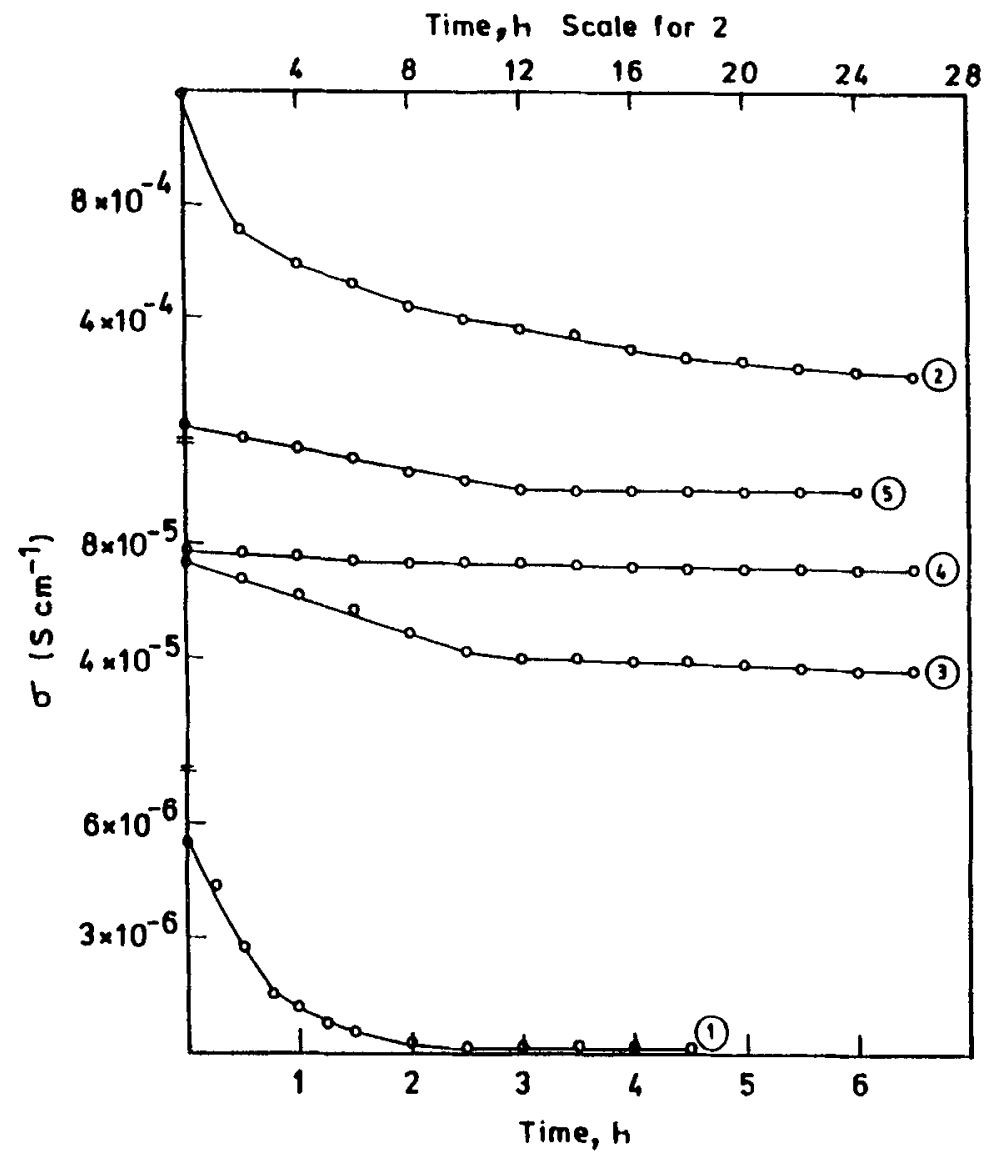

Figure 4. Conductivity as a function of time for benzidine-iodine $(1: 1)(1)$, benzidine-iodine $(1: 1 \cdot 25)(2), o$-tolidine-iodine $(1: 0 \cdot 75)(3), o$-tolidine-iodine $(1: 1)(4)$ and $o$-tolidine-iodine $(1: 1 \cdot 25)(5)$. 


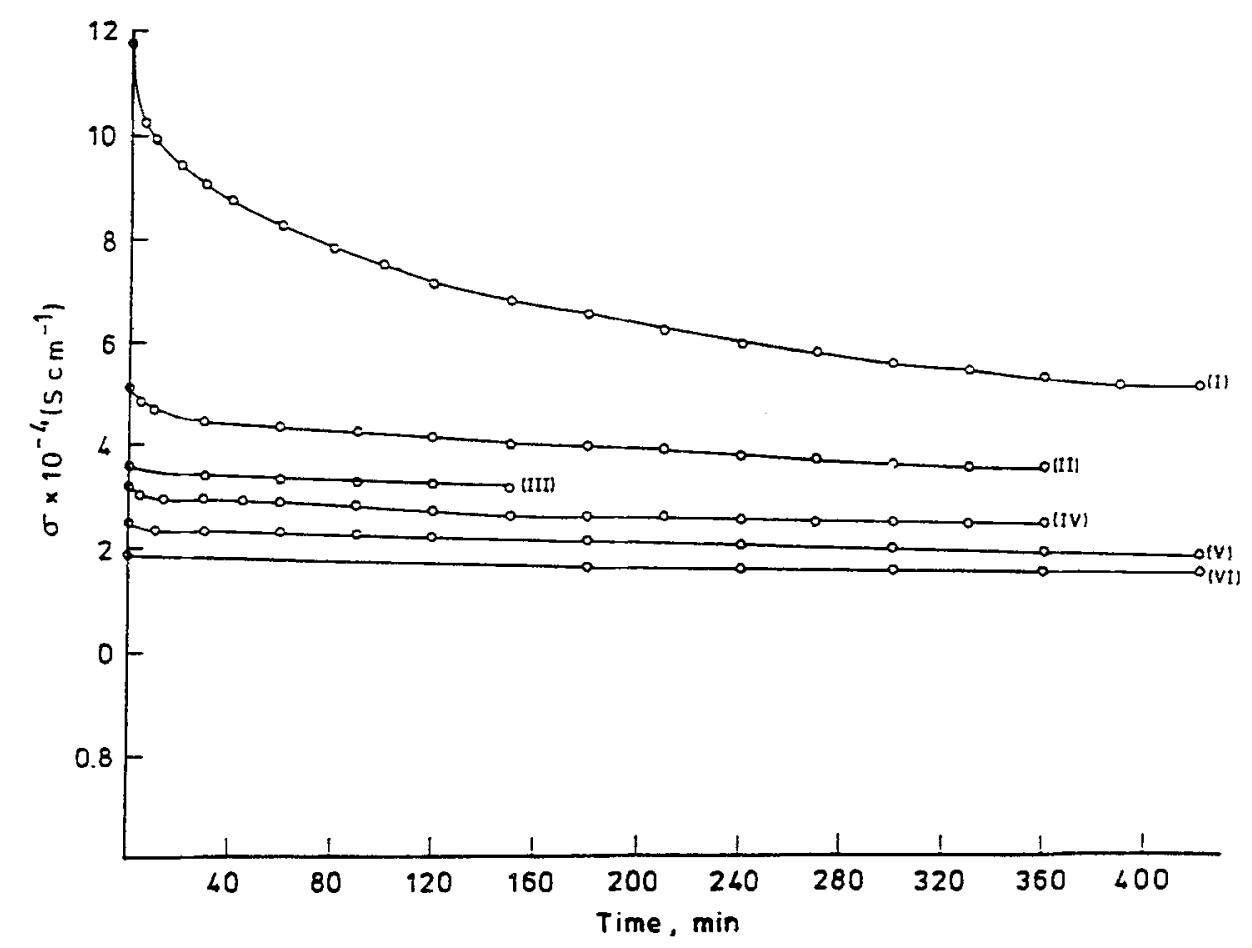

Figure 5. Variation of conductivity as a function of time for benzidine-iodine $(1: 1 \cdot 25)$ complex during electrolysis in different cycles.

Strong $>\mathrm{C}=\mathrm{N}$ around $1670 \mathrm{~cm}^{-1}$ is observed in the cathodic sample; but not in the anodic sample. Also the band around $2370 \mathrm{~cm}^{-1}$ is eliminated in the cathodic sample but it is present as an intense peak in anodic sample just as in the case of pure samples.

\subsection{Current-voltage characteristics}

$I-V$ curves for different compositions were plotted to learn whether the contacts were ohmic or not, and to assess the role of space-charge limited currents. The plot of $I$ vs $V^{2}$ is non-linear, indicating that space-charge limited currents does not play any role in the conduction process at higher voltages. In figure 2 , we report $I-V$ curves for two systems first of benzidine-iodine $(1: 1.25)$ and second of $N$, $N^{\prime}$-diphenylbenzidine-iodine $(1: 1 \cdot 25)$. The curve (1) indicates the existence of a threshold voltage $(2 \mathrm{~V})$ below which the system seems to follow ohmic behaviour but above which it shows change in the slope, indicating enhanced conduction. This is possibly due to onset of ionic conduction. The curve (2) has no such threshold voltage up to $6 \mathrm{~V}$, indicating purely ohmic behaviour and only electronic conduction. However, at still higher voltages, the current falls probably due to discharge of charge carriers at the electrodes. The cyclic $I-V$ curves (figure 3 ) for benzidine-iodine (1:1.25) up to 4 and $10 \mathrm{~V}$ clearly indicate electrolysis, leading to depletion of ionic charge carriers. 


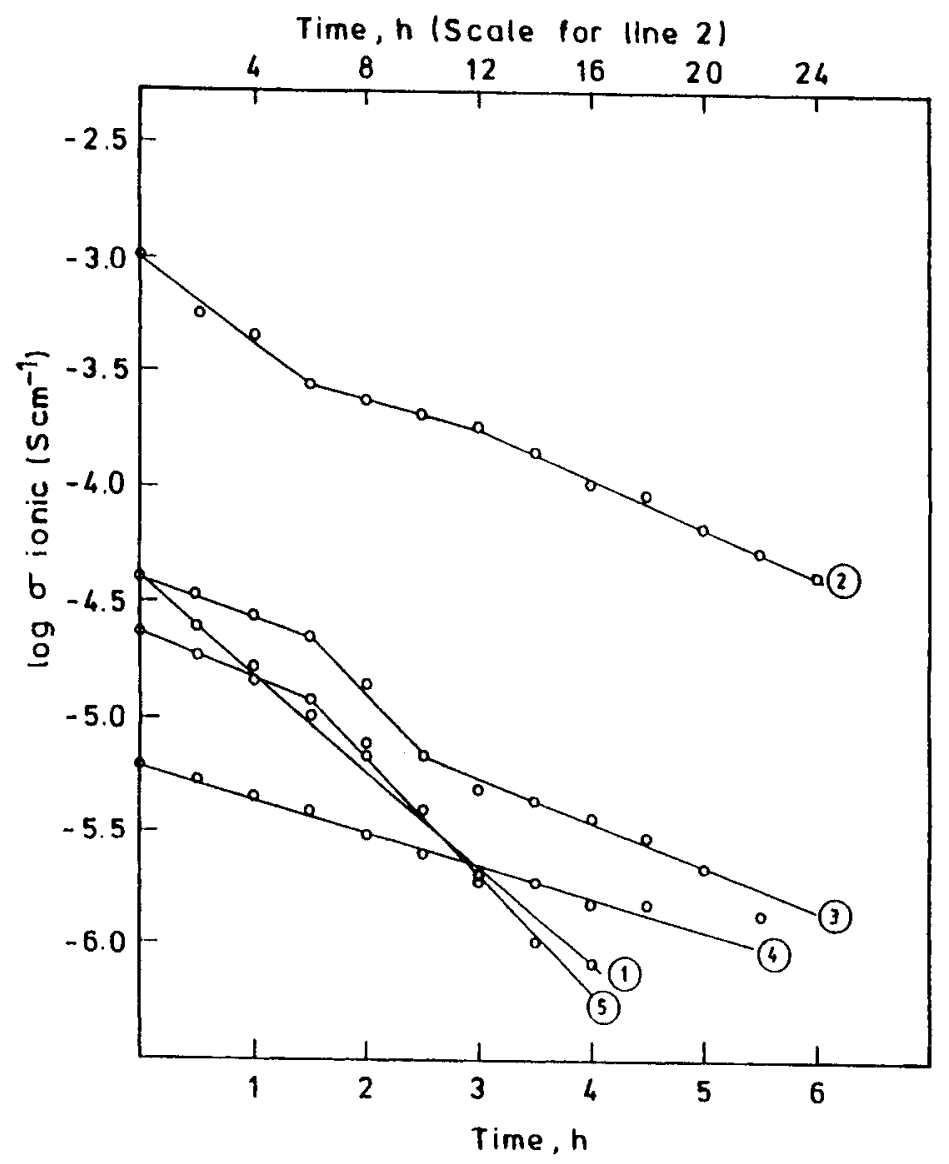

Figure 6. The plot of $\log$ of ionic conductivity as a function of time for benzidine-iodine $(1: 1)$ (1), benzidine-iodine $(1: 1 \cdot 25)(2)$, o-tolidine iodine $(1: 0 \cdot 75)(3), o$-tolidine-iodine $(1: 1)(4)$ and $o$-tolidine-iodine $(1: 1 \cdot 25)(5)$.

\subsection{Time dependence of dc electrical conductivity}

The invariance of electrical conductivity with time has been taken as a measure that most of the charge transfer materials are only electronic conductors rather than ionic ones. However, the existence of mobile ions in materials may lead to some or predominant ionic conduction as in superionic conductors (Chandra 1981). The variation of electrical conductivity with time for different systems has been shown in figure 4. These graphs indicate fast exponential type decrease in electrical conductivity initially, saturating later to almost constant values, which could be separated as electronic $\left(\sigma_{\mathrm{e}}\right)$ and ionic $\left(\sigma_{\mathrm{i}}\right)$ part by extrapolating the linear part to zero time for electronic and point wise subtraction for ionic conduction. The successive electrolysis of benzidine-iodine $(1: 1 \cdot 25)$ over extended period does give constant values of electrical conductivities as shown in figure 5 . It is clear that mobile ions are present in the material and it becomes a purely electronic conductor after a period of electrolysis as reported earlier (Nishizaki et al 1963). Thus, freshly prepared charge transfer complexes of aromatic diamines have mixed ionic-electronic 
Table 1. Electrical data on charge transfer complexes of aromatic diamines with iodine in various compositions obtained by dc study.

\begin{tabular}{|c|c|c|c|c|c|}
\hline \multirow[b]{2}{*}{ Complex } & \multirow{2}{*}{$\begin{array}{c}\text { Conductivity } \\
\text { measured } \\
\text { (literature) } \\
\left(\mathrm{Scm}^{-1}\right)^{*}\end{array}$} & \multicolumn{2}{|c|}{$\begin{array}{l}\text { Thermal } \\
\text { activation } \\
\text { energy } \\
(\mathrm{eV})\end{array}$} & \multirow{2}{*}{$\begin{array}{l}\text { Threshold } \\
\text { voltage } \\
\text { (V) }\end{array}$} & \multirow{2}{*}{$\begin{array}{l}\text { Ionic transpor } \\
\text { number }\end{array}$} \\
\hline & & $\mathrm{Ea}_{1}$ & $\mathrm{Ea}_{2}$ & & \\
\hline \multicolumn{6}{|l|}{$\mathrm{Bz}$-iodine } \\
\hline $1: 0.40$ & $6.6 \times 10^{-7}$ & $0 \cdot 36$ & - & - & - \\
\hline $1: 0.45$ & $8.6 \times 10^{-7}$ & 0.29 & - & - & - \\
\hline $1: 0.75$ & $2.61 \times 10^{-6}$ & $0 \cdot 26$ & - & - & - \\
\hline $1: 1$ & $\begin{array}{c}5.4 \times 10^{-6} \\
\left(1.6 \times 10^{-3}\right)\end{array}$ & $0 \cdot 21$ & - & 10 & 084 \\
\hline $1: 1.25$ & $\begin{array}{c}1.17 \times 10^{-3} \\
\left(4-5 \times 10^{-1}\right)\end{array}$ & 0.08 & - & 2 & $0 \cdot 87$ \\
\hline $1: 1 \cdot 50$ & $4.5 \times 10^{-6}$ & $0 \cdot 11$ & - & - & - \\
\hline \multicolumn{6}{|c|}{$o$-TOL-iodine } \\
\hline $1: 0.75$ & $\begin{array}{c}7-3 \times 10^{-5} \\
\left(2-9 \times 10^{-4}\right)\end{array}$ & 0.23 & $0 \cdot 32$ & 20 & 0.52 \\
\hline $1: 1$ & $\begin{array}{c}7.7 \times 10^{-5} \\
\left(3.4 \times 10^{-3}\right)\end{array}$ & $0 \cdot 11$ & $0 \cdot 32$ & 7 & 0.08 \\
\hline $1: 1 \cdot 25$ & $\begin{array}{c}1.2 \times 10^{-4} \\
\left(3.5 \times 10^{-2}\right)\end{array}$ & 0.085 & 0.21 & 4 & $0 \cdot 20$ \\
\hline \multicolumn{6}{|c|}{ DPBz-iodine } \\
\hline $1: 0.75$ & $2 \cdot 22 \times 10^{-3}$ & $0 \cdot 12$ & - & - & - \\
\hline $1: 1$ & $3 \cdot 32 \times 10^{-3}$ & 0.07 & - & - & - \\
\hline $1: 1 \cdot 25$ & $5-31 \times 10^{-3}$ & 0.013 & - & - & - \\
\hline
\end{tabular}

*Error limit $\pm 0.5 \times 10^{-7} \mathrm{Scm}^{-1}$

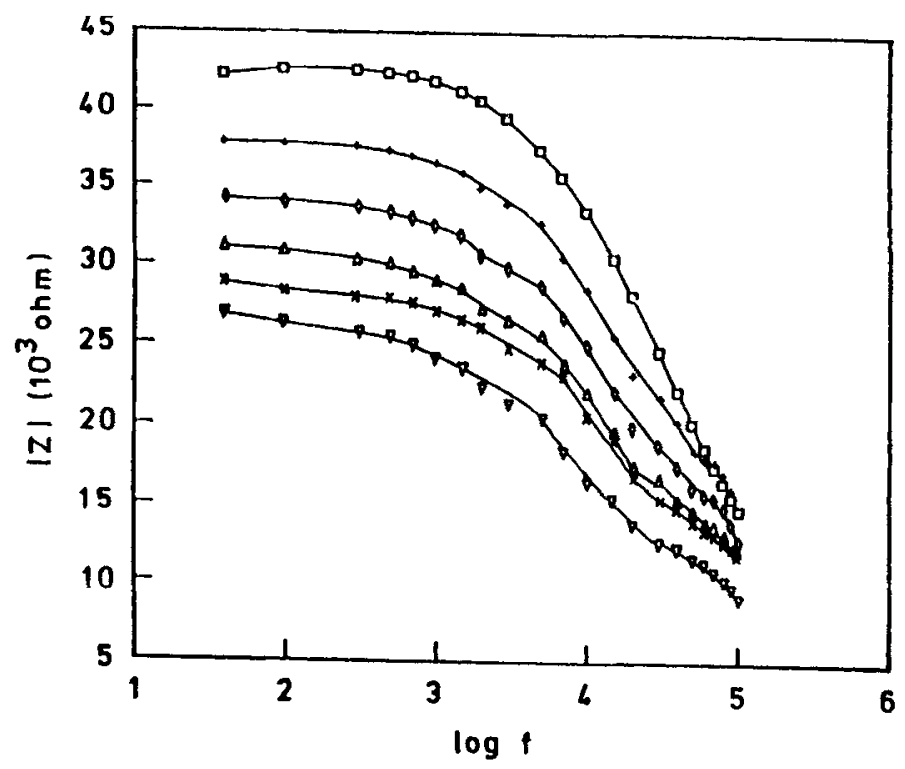

Figure 7. Variation of measured ac impedance $(|Z|)$ with $\log$ of frequency at $30(\square), 40(+)$, $50(\diamond), 60(\Delta), 70(x)$ and $80^{\circ} \mathrm{C}(\nabla)$. 


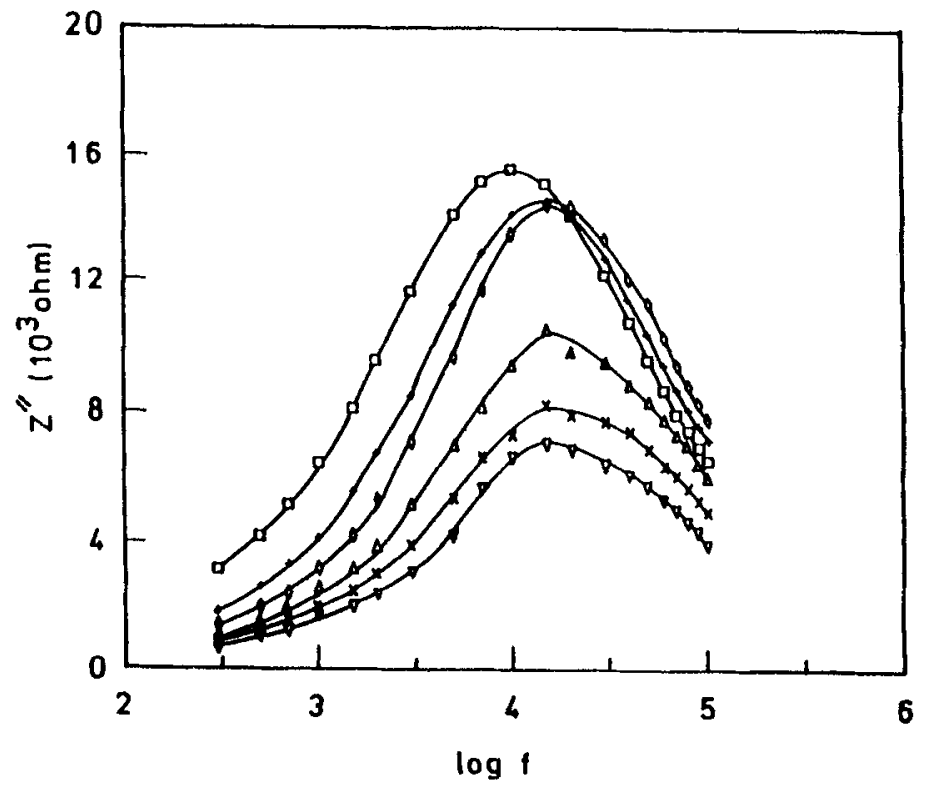

Figure 8. Variation of imaginary impedance $\left(Z^{\prime \prime}\right)$ with $\log f$ at $30(\square), 40(+), 50(\diamond), 60(\Delta)$, $70(\times)$ and $80^{\circ} \mathrm{C}(\nabla)$.

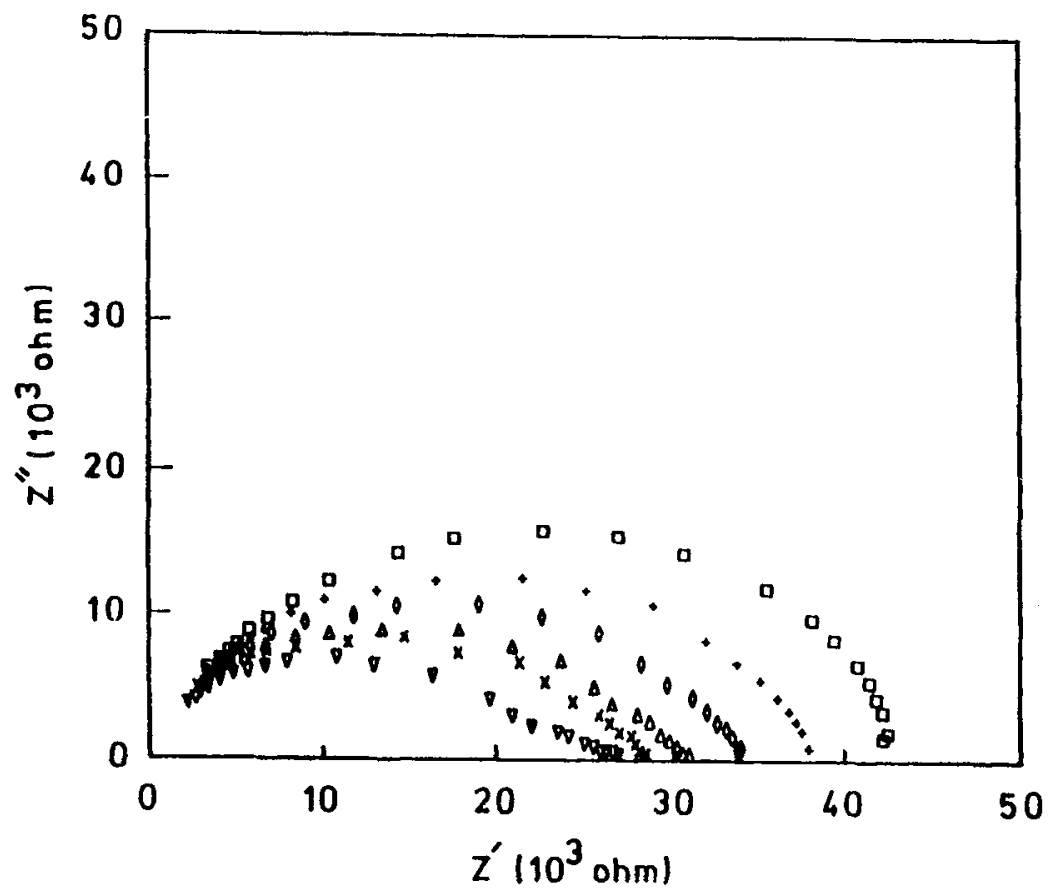

Figure 9. Variation of imaginary impedance $\left(Z^{\prime \prime}\right)$ with real impedance $\left(Z^{\prime}\right)$ at $30(\square), 40(+)$, $50(\diamond), 60(\Delta), 70(x)$ and $80^{\circ} \mathrm{C}(\nabla)$.

conduction. The plot of $\log \sigma_{\mathrm{i}}$ vs time shown in figure 6 yields the electromigration rate constants for different systems. Different dc parameters have been given in table 1 . 
Table 2. Electrical data on charge transfer complexes of aromatic diamine with iodine in various compositions obtained by ac study.

\begin{tabular}{|c|c|c|c|c|c|}
\hline Complex & $\begin{array}{c}\text { Conductivity } \\
\text { measured } \\
\left(\mathrm{Scm}^{-1}\right)^{*}\end{array}$ & $\begin{array}{l}\text { Capacitance } \\
(\mathrm{nF})^{* *}\end{array}$ & $\begin{array}{c}\text { Capacitance } \\
(\mathrm{pF})^{* * *}\end{array}$ & $\begin{array}{c}\text { Dielectric } \\
\text { constant } \\
\left(\mathrm{K}=\mathrm{C} / \mathrm{C}_{0}\right)^{* *}\end{array}$ & $\begin{array}{l}\text { Dissipation } \\
\text { factor** }\end{array}$ \\
\hline \multicolumn{6}{|l|}{ Bz-iodine } \\
\hline $1: 0.75$ & $1.46 \times 10^{-6}$ & $0 \cdot 30$ & 34.5 & $4.8 \times 10^{2}$ & $16 \cdot 10$ \\
\hline $1: 1$ & $3.51 \times 10^{-5}$ & $4 \cdot 60$ & 147.0 & $7.2 \times 10^{3}$ & $76-55$ \\
\hline $\begin{array}{l}1: 1 \cdot 25 \\
\text { o-TOL-io }\end{array}$ & $1.17 \times 10^{-3}$ & 1.67 & $180 \cdot 0$ & $2 \cdot 3 \times 10^{3}$ & 76.55 \\
\hline $1: 0.75$ & $1.06 \times 10^{-5}$ & $0 \cdot 3$ & $70 \cdot 8$ & $2.66 \times 10^{2}$ & $760 \cdot 00$ \\
\hline $1: 1$ & $5.42 \times 10^{-5}$ & $0 \cdot 1$ & $92 \cdot 4$ & $1.3 \times 10^{2}$ & $8854 \cdot 00$ \\
\hline $1: 1.25$ & $7.16 \times 10^{-4}$ & 198.0 & $210 \cdot 0$ & $3.69 \times 10^{5}$ & 15.73 \\
\hline \multicolumn{6}{|c|}{ DPBz-iodine } \\
\hline $1: 0.75$ & $2 \cdot 30 \times 10^{-3}$ & $50 \cdot 0$ & $2550 \cdot 0$ & $5.4 \times 10^{4}$ & $1223 \cdot 7$ \\
\hline $1: 1$ & $3.90 \times 10^{-3}$ & - & - & - & $13475-0$ \\
\hline $1: 1.25$ & $6.28 \times 10^{-3}$ & - & - & - & $13307 \cdot 0$ \\
\hline
\end{tabular}

*Values determined from the bulk resistance by CNLS fit.

** Values measured at $100 \mathrm{~Hz}$ frequency.

*** Values measured at $100 \mathrm{KHz}$ frequency.

Out of instrument measurement range due to very high values.

\subsection{AC impedance study}

The AC impedance studies on different systems as a function of frequency and temperature have been made. A representative example for benzidine-iodine $(1: 1 \cdot 25)$ using platinum contacts have been given in figures 7-9. The measured impedance as a function of frequency at different temperatures for this system is shown in figure 7. It is evident from this figure that the impedance decreases as a function of frequency and temperature which may be due to semiconductive nature of the material as well as shorting out of ionic contribution at high frequencies. The plot of $Z^{\prime \prime}$ vs $\log f$ at different temperatures shown in figure 8 have maxima shifting to lower values at higher temperatures from which the conductivities of samples have been evaluated using relevant equations. The plot of $Z^{\prime \prime}$ vs $Z^{\prime}$ in figure 9 shows marked changes with temperature, both in shape and magnitude of circuit elements. These graphs were fitted in (RC) (RC) equivalent circuits using complex non-linear least square (CNLS) fitting software (EG \& G). The different AC impedance parameters have been reported in table 2. The Arrhenius plots at lower frequencies have single slopes giving thermal activation energies comparable to those by dc values but the bulk conductivity values obtained from the plots of $Z^{\prime \prime}$ vs $\log f$, yield two slopes, one giving the values corresponding to $\mathrm{dc}$ method and the other of the order of $0.25 \mathrm{eV}$, which may be the thermal activation energy for ionic conduction.

\subsection{Transport numbers}

The relative magnitude of ionic conductivity to electronic conductivity was determined by the extrapolation of linear portion and subtracting point-wise. The diffusion 
parameter for electromigration of ions was determined from the plot of logarithm of ionic conductivity against time as shown in figure 6 . The derived parameters have been reported in table 1 . These data indicate that in $1: 1.25$ benzidine-iodine complex, the ionic transport number $\left(t_{\mathrm{i}}\right)$ determined from the ratio of $\sigma_{\mathrm{i}} / \sigma_{\text {total }}$ has a value of 0.87 , almost equal to superionic proton conductors (Chandra 1981). However, the most conducting ratio of $\mathrm{Bz}-\mathrm{I}_{2}(1: 1 \cdot 25)$ has lower threshold voltage (cf table 1$)$.

$o$-Tolidine-iodine complexes have been found to have much lower values of ionic transport number but the presence of ionic conduction has not been ruled out. The lower values may be attributed to some other ionic carrier such as halides, although its existence could not be ascertained. The dc conductivity of $N, N^{\prime}$-diphenylbenzidineiodine shows time-independent nature, indicating that this system may be purely electronic conductor.

\section{Conclusion}

The data presented in this paper, clearly indicate mixed conduction (ionic-electronic) behaviour of some charge transfer complexes of iodine with benzidine and $o$-tolidine. The formation of mobile ionic species such as proton at $-\mathrm{N}^{+} \mathrm{H}$ may be responsible for the ionic contributions. A possible mechanism of the formation of ionic charge carriers and different electrode reactions during electrolysis has been proposed.

\section{Acknowledgements}

Financial assistance from the Department of Science and Technology, New Delhi and University Grants Commission, New Delhi are gratefully acknowledged. Authors thank the referees for their comments.

\section{References}

Akamatu H, Inokuchi $\mathrm{H}$ and Matsunaga Y 1954 Nature 173168

Akamatu H, Inokuchi $\mathrm{H}$ and Matsunaga Y $1956 \mathrm{~J}$. Am. Chem. Soc. 29214

Ashwell G J 1992 Molecular electronics (England: Research Studies Press Ltd.)

Chandra S 1981 Superionic solids (Amsterdam: North Holland)

Gutmann F and Lyons L E 1967 Organic semiconductors (New York: John Wiley and Sons Inc.)

Kommandeur J and Hall F R 1961 J. Chem. Phys. 34129

Kusabayashi S, Mikawa H, Kawai S, Uchida M and Kiriyama R 1964 Bull. Chem. Soc. Jpn 37811

Naik H M and Subramanyam S V 1986 Pramana -J. Phys. 26 61-66

Nisizaki S and Kusakawa H 1963 Bull. Chem. Soc. Jpn 361681

Nisizaki S and Kusakawa H 1965 Bull. Chem. Soc. Jpn 38313

Ravindran T and Subramanyam S V 1991a Bull. Mater. Sci. 141205

Ravindran T and Subramanyam S V 1991b Pramana-J. Phys. 371991

Sakai H, Muraishi S and Maeda Y 1987 Bull. Chem. Soc. Jpn. 60857

Singh R A. Singh R and Verma S M 1991 Bull. Chem. Soc. Jpn. 641938

Singh R A, Rao O S and Singh V K 1996a Ind. J. Engg. Mat. Sci. 323

Singh V K, Rao O S and Singh R A 1996b Ind. J. Engg. Mat. Sci. 3201

Weinberg N L 1974 Technique of electroorganic synthesis (New York: John Wiley and Sons Inc.) 